\title{
Morphometric Analysis of The Accessory Transverse Foramen in a Population in Northeastern Brazil
}

\author{
João Pedro Maia Medeiros, ${ }^{1}$ Jamil Dieb Santana, ${ }^{1}$ Fábio Antônio Serra de Lima Júnior, ${ }^{1}$ Jonathan Barros Cavalcante, ${ }^{2}$ Cecília \\ de Lima Leite, 2 Sandy Costa Gonçalves, ${ }^{2}$ Gilberto Santos Cerqueira, ${ }^{2}$ Desiré Dominique Diniz de Magalhães, ${ }^{1}$ Jalles Dantas \\ de Lucena, ${ }^{3}$ André de Sá Braga Oliveira ${ }^{1}$
}

${ }^{1}$ Federal University of Paraiba - UFPB. João Pessoa, PB, Brazil

${ }^{2}$ Federal University of Ceara - UFC. Fortaleza, CE, Brazil

${ }^{3}$ Faculty Santa Maria- FSM. Cajazeiras, PB, Brazil

Disclose and conflicts of interest: none to be declared by all authors

\begin{abstract}
Introduction: vertebral arteries (VA) are the formation factors of the Transverse Foramens (TF) and the largerst anatomical structures that occupy them. Variations in the presence, size and course of VAs affect the morphology of TFs considerably, besides being the probable cause of formation of the accessory transverse foramen (ATF). Knowledge of the presence of the ATF and its variations are important for a safe surgery.

Material and Methods: the present study has analyzed 165 cervical vertebrae. The ATF has been classified as unilateral or bilateral, complete or incomplete and anterior, posterior or lateral regarding the transverse foramen of the respective cervical vertebrae analyzed. Quantitative data was collected through a digital caliper.

Results: $36(21.82 \%)$ of the vertebrae presented the ATF, with $25(15.15 \%)$ of them carrying the ATF unilaterally, while the remaining 11 (6.67\%) had the foramen bilaterally. 28 (59.57\%) ATF were classified as incomplete and 19 (40.43\%) were classified as complete. 44 (93.62\%) were identified as posterior and $3(6.38 \%)$ were anterior to the TF. Regarding the morphometric data, there was no difference between the longitudinal measurement (LM) and transverse measurement (TM) on the right and left sides of the vertebrae analyzed.

Conclusion: it has been shown for the first time the prevalence of ATF in individuals in a population of Northeastern Brazil. The morphological knowledge can be surgical and clinically important as it may suggest alterations in the vertebral artery and in the venous plexuses that surround it in its vertebral segment of passage in the transverse foramina.

Keywords: Anatomy; Clinical anatomy; Spine; Transverse foramen.
\end{abstract}

\section{Introduction}

The transverse foramen (TFs) are located bilaterally in the transverse processes of the cervical vertebrae, which are the smallest among the mobile vertebrae. They are filled by the spiral path of the vertebral arteries (AVs). ${ }^{1}$

The vertebral artery, which comes up at the initial portions of the subclavian arteries, medially to the anterior scalene muscle, can be divided into four segments: cervical, vertebral, suboccipital and intracranial. The vertebral portion starts with the penetration of the vessel in the TF of the sixth cervical vertebra in an ascending path to the atlas ( $\mathrm{C} 1$ vertebrae). Next to the roots of the spinal nerves there is the emergence of spinal branches of this artery, which go towards the spinal cord and contribute to its irrigation. It is noteworthy that in this segment the VA is surrounded by a venous plexus. ${ }^{2}$
Thus, the anatomical relations described in this region explain why during prolonged head rotations, such as when driving in reverse, there may be dizziness and other symptoms, since there is interference in the blood supply of the brainstem that comes from branches of the VA.

The vertebral arteries are the factors that form FTs, and the largest anatomical structures that occupy them. In about $88 \%$ of cases, VA can enter the cervical spine through the sixth vertebra, but occasionally it can emerge in the fourth, fifth or seventh cervical vertebra. ${ }^{3}$ In the 7 th cervical vertebra, the foramen is smaller and give way to only small accessory vertebral veins, which are sometimes absent. Variations in the presence, size and course of the VAs affect the morphology of the TFs considerably, in addition to being the probable cause of formation of the accessory transverse foramen (ATF). 
The accessory transverse foramen (ATF) or double transverse foramen, according to some publications, is characterized by being a considerably smaller structure than the TF, and may be absent in most individuals. It is also located in the transverse processes of the cervical vertebrae and is defined by its spatial relationships with the TF of the respective vertebra. Unlike the TF, it can appear due to changes in venous circulation in the region. Knowledge of the presence of the ATF and its variations are important for a safe surgery. ${ }^{4,5}$ Changes in the embryological process of formation of vertebral vessels are the most suggestive causes of the formation of ATF. This relation is corroborated to the extent that, as reported in most of the literature on the topic, the presence of ATF is more common in low cervical vertebrae (C5, C6, C7), especially at the exact C6 level where there is penetration of the VA in most individuals. ${ }^{6}$

\section{Material and Methods}

The present study has analyzed 165 dry cervical vertebrae from a population of Northeastern Brazil, with unknown ages and genders, belonging to the morphology department, more specifically to the human anatomy laboratories at the Federal University of Paraíba (UFPB). Vertebrae with important degradation were excluded from the study, once they made their specific analysis impossible.

As qualitative parameters, the accessory transverse foramina have been classified as unilateral or bilateral, complete or incomplete, and anterior, posterior or lateral, in relation to the transverse foramen of the respective cervical vertebrae analyzed (Figure 1).

A $150 \mathrm{~mm}$ capacity digital caliper (Eccofer ${ }^{\circledR}$, João Pessoa, Paraíba, Brazil) was used to collect the morphometric data of the accessory transverse foramen, including the verifications of the longitudinal measurement (LM) and transverse measurement (TM) of the diameters of the ATF. For proper analysis of these data, the Kolmogorov-Smirnov test was used to verify its normal distribution and, immediately afterwards, parametric or non-parametric tests were applied to assess the difference between the right and left sides of the analyzed morphometric parameters. The results were considered significant when $\mathrm{p} \leq 0.05$.

\section{Results}

As shown by the analysis of the 165 vertebrae present in the study, we have identified the presence of $44(26.67 \%)$ atlas vertebrae, 58 (35.15\%) axis vertebrae and 63 (38.18\%) typical cervical vertebrae (C3-C7). Among them, two axis vertebrae were excluded from the study because they had damaged structures that compromised the analysis.

Segmenting the analysis by vertebrae type, 4 (2.43\%) atlas vertebrae with the ATF were identified, while $40(24.24 \%)$ did not have this structure. Moreover, $8(4.84 \%)$ axis vertebrae presented the ATF, while 48 (29.09\%) did not have it and 2 were damaged (1.21\%). Finally, 24 (14.54\%) of the typical cervical vertebrae were identified with this foramen, with the remaining 39 (23.64\%) were identified without this structure (Table 1).

Regarding the presence of the accessory transverse foramen, $36(21.82 \%)$ of the vertebrae presented this structure, with 25 (15.15\%) of them having unilateral ATF, while the remaining 11 (6.67\%) had the foramen bilaterally. Thus, a total number of 47 accessory transverse foramen was found in the vertebrae included in the study.

Furthermore, according to the qualitative analysis

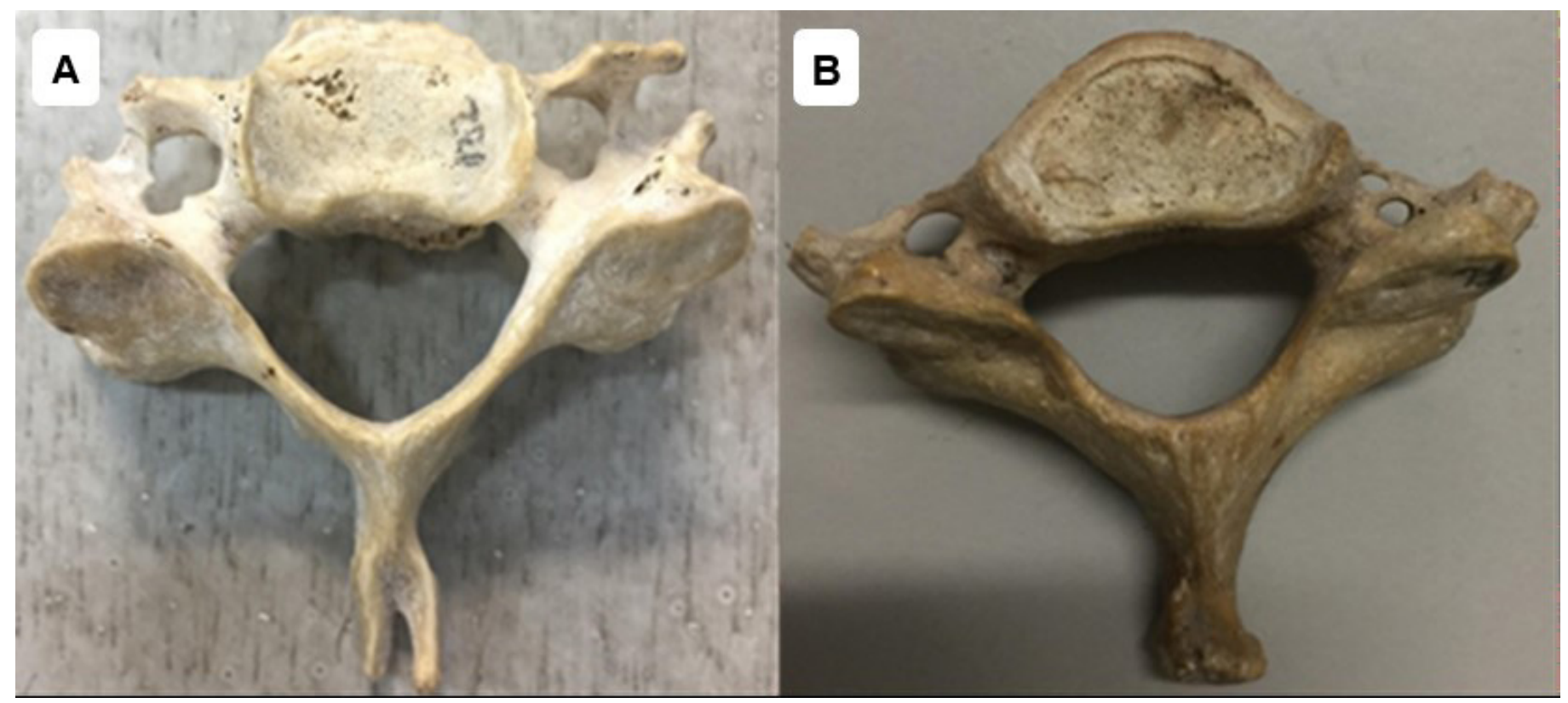

Figure 1. Qualitative analysis of the accessory transverse foramen: (A) ATF bilateral, incomplete and posterior, (B) ATF unilateral, complete and anterior. 
of the 47 accessory accessory foramens identified, 28 (59.57\%) were classified as incomplete and 19 (40.43\%) as complete. According to the anatomical relationship with the transverse foramen, 44 (93.62\%) were identified as posterior and $3(6.38 \%)$ as anterior to this other prominent structure. No ATF was identified in a lateral position to the TF (Table 2).

Finally, as shown in the linear dimensions of the ATF analyzed, the mean transverse diameter was $3.11 \pm 0.51$ $\mathrm{mm}$ (range 1.86 to $4.30 \mathrm{~mm}$ ) and $3.13 \pm 0.52 \mathrm{~mm}$ (range 1.84 to $4.30 \mathrm{~mm}$ ) on the right and left, respectively. The longitudinal diameter was $2.49 \pm 0.41 \mathrm{~mm}$ (range from 1.90 to $3.47 \mathrm{~mm}$ ) and $2.47 \pm 0.49 \mathrm{~mm}$ (range from 1.59 to $3.45 \mathrm{~mm}$ ), also on the right and left sides, respectively. There were no differences between the linear parameters verified in the accessory transverse foramen between two sides of the vertebrae $(p<0.05)$ (Table 3). of the ATF in dry cervical vertebrae in a population in Northeastern Brazil.

The presence of the accessory transverse foramen was reported in 36 of the 165 vertebrae analyzed, corresponding to a percentage of $21.82 \%$. The values presented are similar to those described by Esakkiammal and Chauhan $(2016)^{7}$, who reported the presence of 37 (27.6\%) ATF in 134 analyzed vertebrae. Only simple cervical vertebrae were considered by this study, since the analysis did not identify the presence of ATF in $\mathrm{C} 1$ or $\mathrm{C} 2$. In addition, Chaudhari et al. $(2013)^{1}$ also described a prevalence of $31(23.15 \%)$ in 133 vertebrae analyzed, thus bringing an analysis close to that identified in this study.

On the other hand, Murlimanju et al. (2011) ${ }^{8}$ described the presence of only 6 (1.6\%) ATF in 363 vertebrae analyzed, contrasting with the proportion analyzed in the present study. Also, Murlimanju et al.

Table 1. Distribution of the Accessory Transverse foramen (ATF) in the analyzed vertebrae $(\mathrm{N}=165)$.

\begin{tabular}{c|c|c|c}
\hline Vertebrae & With FTA & Without FTA & Total \\
\hline Atlas & $4(2.43 \%)$ & $40(24.24 \%)$ & $44(26.67 \%)$ \\
\hline Axis & $8(4.84 \%)$ & $48(29.09 \%)$ & $58(35.15 \%)$ \\
\hline Typical (C3-C7) & $24(14.54 \%)$ & $39(23.64 \%)$ & $63(38.18 \%)$ \\
\hline Total & $36(21.82 \%)$ & $129(78.18 \%)$ & $165(100 \%)$ \\
\hline
\end{tabular}

Table 2. Qualitative analysis of ATF laterality, shape and position. $\mathrm{N}=47$.

\begin{tabular}{l|c|c|c|c|c|c}
\hline \multicolumn{2}{c|}{ Laterality } & \multicolumn{2}{c}{ Shape } & \multicolumn{2}{c}{ Position } \\
\hline Unilateral & Bilateral & Incomplete & Complete & Anterior & Posterior & Lateral \\
\hline $25(15.1 \%)$ & $11(6.7 \%)$ & $28(59.6 \%)$ & $19(40.4 \%)$ & $3(6.4 \%)$ & $44(93.6 \%)$ & - \\
\hline
\end{tabular}

Table 3. Mean $(\mathrm{mm}) \pm$ standard deviation (SD) values of the morphometric parameters analyzed in the accessory transverse foramen (ATF) of the cervical vertebrae. $\mathrm{N}=47$.

\begin{tabular}{c|c|c|c|c}
\hline & \multicolumn{2}{|c|}{ Right side } & \multicolumn{2}{c}{ Left side } \\
\hline Parameters & Min - Max & Mean (SD) & Mean (SD) \\
\hline TD & $1.86-4.30$ & $3.11 \pm 0.51$ & $1.84-4.30$ & $1.13 \pm 0.52$ \\
\hline LD & $1.59-3.47$ & $2.49 \pm 0.41$ & $2.47 \pm 0.49$ & \\
\hline
\end{tabular}

TD: Transverse diameter of ATF; LD: longitudinal diameter of ATF; N: number; Min: Minimum; Max: Maximum; SD: Standard Deviation.

\section{Discussion}

Changes in the embryological process of vessel formation in the cervical region are the main causes of the appearance of accessory transverse foramen. Although there is still no consensus regarding its clinical manifestations, it is extremely important that its morphological knowledge is analyzed in surgical approaches, as to fix vertebrae fractures, for example, and imaging exams in the region.

Hence, believing in the importance of identifying morphometric distinctions between epidemiological groups analyzed, enabling a more precise knowledge for the professionals working with this specific area of the body, the present study analyzed the prevalence
$(2011)^{8}$ described the presence of ATF only on cervical vertebrae. In this same perspective, Das S et al. (2005) ${ }^{9}$ published a study in which the presence of the ATF was described in 2 (1.5\%) of 132 vertebrae analyzed. Sharma et al. $(2010)^{10}$ described the presence of 16 (8\%) ATF in 200 vertebrae, notably at the C6 level. Finally, Taitz et al. $(1978)^{11}$ used a sample space of 480 vertebrae, in which the ATF was identified in only 34 (7\%) (Table 4).

This literature review on the subject has shown a higher prevalence of the ATF in the vertebrae of this study in contrast to a considerable part of the existing publications, although some authors demonstrate similar values. ${ }^{1,7}$ It is notorious, however, to emphasize that there are very few reports about the presence 
of ATF in atlas or axis vertebrae ( $\mathrm{C} 1$ and $\mathrm{C} 2$ levels, respectively), with the descriptions being very restricted to typical cervical vertebrae only.

Esakkiammal and Chauhan (2016) ${ }^{7}$ and Murlimanju et al. $(2011)^{8}$ published that the presence of ATF was only described in typical cervical vertebrae, whereas Akhtar et al. (2015) ${ }^{12}$ cites the presence of ATF in 9 atypical vertebrae. However, the author does not mention any axis vertebrae on his report with this structure. In contrast, this present study identified $4 \mathrm{C} 1$ vertebrae and $8 \mathrm{C} 2$ vertebrae that had the ATF. Although it is still mostly described in typical cervical vertebrae ( 24 in the present study), the fact that the ATF is identified in atypical cervical vertebrae, both $\mathrm{C} 1$ and $\mathrm{C} 2$ is a highly innovative finding in the area.

Additionally, Akhtar et al. (2015) ${ }^{12}$ describes 20 unilateral and 5 bilateral ATFs, just like Gonsai et al. $(2015)^{13}$ does, identifying 31 unilateral and 9 bilateral, being in correspondence with the findings in the present study (25 vertebrae with unilateral ATF and 11 with bilateral ATF). On the other hand, Esakkiammal and Chauhan (2016) ${ }^{7}$ and Mishra et al. (2015) ${ }^{14}$ reported a more frequent bilaterality in their analysis, with 18 unilateral and 19 bilateral for the first and 10 unilateral and 21 bilateral for the second one (Table 5).

Other studies have also classified their analysis as to whether the ATF is complete or incomplete. The prevalence identified by Mishra et al. $(2015)^{14}$ consisted of 24 complete ATFs against only 7 in their incomplete morphology. Such data are correspondent with the description of Gonsai et al. $(2015)^{13}$, whose sample presented 40 vertebrae that had the ATF in complete shape, while only 22 were incomplete, and with the analysis of Kumari et al. (2015) ${ }^{15}$, in which 22 ATF were complete and only 9 were incomplete.

These data contrast with those identified by Esakkiammal and Chauhan (2016) ${ }^{7}$, who described the prevalence of 19 complete morphology ATF and 24 in its incomplete presentation, and with those exposed in the present analysis, since it has described only 19 ATF in its complete morphology and 28 of the accessory transverse foramen as being incomplete. Therefore, the innovative character of the study is again perceived, as it brings relevant information with divergent outcomes in the literature (Table 6).

Finally, the quantitative analysis described in the results of the present study is more complete than the existing records in the literature, considering that the articles that mention the size of the ATF do so in a brief way, only highlighting the fact of presenting smaller diameters than the foramen transverse itself, without going deeper into the measurements made and described here previously, with emphasis on the fact that there is no differences between the right and left sides of ATF.

Table 4. Comparison of the percentage of ATF in different populations.

\begin{tabular}{|c|c|c|}
\hline Population & Total Vertebrae & Vertebrae with ATF \\
\hline Taitz et al. $1978,{ }^{11}$ Tel-Aviv, Israel & 480 & $34(7 \%)$ \\
\hline Das S et al. $2005,{ }^{9}$ Bangi, Malaysia & 132 & $2(1.5 \%)$ \\
\hline Sharma et al. $2010,^{10}$ India & 200 & $16(8 \%)$ \\
\hline Murlimanju et al. $2011,{ }^{8}$ India & 363 & $6(1.6 \%)$ \\
\hline Chaudhari et al. 2013, Bhuj, India & 133 & $31(23.15 \%)$ \\
\hline Esakkiammal and Chauhan. 2016,7 Delhi, India & 134 & $37(27.6 \%)$ \\
\hline Present study & 165 & $36(21.82 \%)$ \\
\hline
\end{tabular}

Table 5. Comparative analysis of ATF laterality.

\begin{tabular}{|c|c|c|}
\hline Population & Unilateral ATF & Bilateral ATF \\
\hline Akhtar et al. $2015,{ }^{12}$ India & 20 & 5 \\
\hline Gonsai et al. 2015,13 Ahmedabad, India & 31 & 9 \\
\hline Mishra et al. 2015,14 Lucknow, India & 10 & 21 \\
\hline Esakkiammal and Chauhan, 2016, ${ }^{7}$ Delhi, India & 18 & 19 \\
\hline Present study & 25 & 11 \\
\hline
\end{tabular}

Table 6. Comparative analysis of ATF shape.

\begin{tabular}{|c|c|c|}
\hline Population & Complete ATF & Incomplete ATF \\
\hline Gonsai et al. 2015,13 Ahmedabad, India & 40 & 22 \\
\hline Mishra et al. 2015, ${ }^{14}$ Lucknow, India & 24 & 7 \\
\hline Kumari et al. 2015,15 Kishanganj, India & 22 & 09 \\
\hline Esakkiammal and Chauhan, 2016,7 Delhi, India & 19 & 24 \\
\hline Present study & 19 & 28 \\
\hline
\end{tabular}




\section{Conclusion}

For the first time, the prevalence of the accessory transverse foramen was demonstrated in a population in Northeastern Brazil, along with a complete analysis of the morphometric parameters of this structure, such as location in relation to the transverse foramen, unilateral or bilateral character and complete or incomplete shape. The presence of the accessory transverse foramen was observed in $21.82 \%$ of the cases, with the unilateral presence being more frequent than the bilateral and all cervical vertebrae (Atlas, Axis and Typical $\mathrm{C} 3$ to $\mathrm{C} 7$ ) being affected by this variation. Incomplete morphology prevailed and the posterior relationship with FT was more frequent. The study found no significant differences in transverse and longitudinal diameters when laterality was considered.

These identifications contrasted to several findings in the literature, such as the higher prevalence of the ATF in this analysis, evidencing the innovative character of the study, bringing significant contributions to the anatomy field in Brazil and serving as a basis for neurosurgeons, head and neck surgeons, orthopedists, radiologists and physiotherapists. Morphological knowledge is clinically important, as it can suggest changes in the vertebral artery and in the venous plexuses that surround it in its vertebral segment passing through the transverse foramen.

The presence of the accessory transverse foramina may suggest fenestrations or duplications in the vertebral artery or in important veins. Variations in their location, shape and quantity can increase the risk of injury to these vessels during surgical access to the cervical spine, which can produce clinical complications and iatrogenic injuries. Finally, compression of the vertebral artery during neck movement can cause basilar failure, characterized by headache, fainting and hearing problems.

\section{References}

1. Chaudhari ML, Maheria PB, Bachuwar SP. Double foramen transversarium in cervical vertebra. Morphology and clinical importance. Indian J Basic Appl Med Res 2013;8(2):1084-1088.

2. Meneses MS. Neuroanatomia aplicada. Rio de Janeiro, Guanabara Koogan, 2011. Cap 23, p 299.

3. Standring S. Gray's Anatomia: A base anatômica da prática clínica. 40a edição. Rio de Janeiro, Elsevier, 2010.

4. Le Minor JM. The retrotransverse foramen of the human atlas vertebra. A distinctive variant within primates. Acta Anat (Basel);160:208-212.

5. Le Minor J-M, Trost O. Bony ponticles of the atlas (C1) over the groove for the vertebral artery in humans and primates: Polymorphism and evolutionary trends. Am J Phys Anthropol 2004;125(1):16-29.

6. Gupta S, Patel Z, Gautam RS. Morphological Study of Acessory Foramen Transversarium in Dried Cervical Vertebrae in Human Being. Int J Anat Res 2017;5(2.2):3791-3795.

7. Esakkiammal N, Chauhan R. Clinical significance of presence of accessory foramen transversarium in typical cervical vertebrae. Int J Res Med Sci 2016;4:5231-5236.

8. Murlimanju BV, Prabhu LV, Shilpa K, Rai R, Dhananjaya KVN, Jiji PJ. Accessory transverse foramina in the cervical spine: incidence, embryological basis, morphology and surgical importance. Turk
Neurosurg 2011;21(3):384-387.

9. Das S, Suri RK, Kapur VK. Double foramen transversaria: An osteological study with clinical implications. Int Med J 2005;12(4):311-313.

10. Sharma A, Singh K, Gupta V, Stivastava S. Double foramen transversarium in cervical vertebra na osteological study. J Anat Soc India 2010;59(2):229-231.

11. Taitz C, Nathan H, Arensburg B. Anatomical observations of the foramina transversaria. J Neurol Neurosurg Psychiatry 1978;41(2):170176.

12. Akhtar MJ, Madhukar PK, Rahman S, Kashyap N. A morphometric study of foramen transversarium of dried cervical vertebrae. Int J Res Med Sci 2015;3(4):912-916.

13. Gonsai V, Jadav J, Shah HR. Variations in Transverse Foramina of Cervical Vertebrae: Morphology \& Clinical Importance. BJKinesNJBAS 2015;7(2):6-9.

14. Mishra GP, Kumari S, Bhatnagar S, Singh B. Sixth cervical vertebra with bilateral double foramen transversarium and non-bifid spine: a rare case. Int J Res Med Sci 2015;3(1):352-353.

15. Kumari M, Omar S, Deb S, Alam K. An Osteological Study on Accessory Transverse Foramina in Cervical Vertebrae and Their Clinical Significance. Int J Rec Sci Res 2015:6(6):4514-4516.
Received: January 30, 2019

Accepted: July 25, 2020
Corresponding author

André de Sá Braga Oliveira

andre.sboliveira@gmail.com 